\title{
Association Between Halitosis Diagnosed by a Questionnaire and Halimeter and Symptoms of Gastroesophageal Reflux Disease
}

\author{
Hyo-Jung Lee, ${ }^{1}$ Hee Man Kim, ${ }^{2}$ Nayoung Kim, ${ }^{3,4 *}$ Jane $\mathrm{C} \mathrm{Oh}^{5}$ Hyun Jin Jo, ${ }^{3}$ Jung-Tae Lee, ${ }^{1}$ Hee-Yung Chang, ${ }^{6}$ Na-Hee Chang, ${ }^{7,8}$ \\ Soyeon $\mathrm{Ahn}^{9}$ and Jeong-Yun Lee ${ }^{10}$ \\ ${ }^{1}$ Departments of Periodontology, Section of Dentistry and ${ }^{3}$ Internal Medicine, Seoul National University Bundang Hospital, Seoungnam, \\ Gyeonggi-do, Korea; ' Department of Internal Medicine, Yonsei University Wonju College of Medicine, Wonju, Gangwon-do, Korea; \\ ${ }^{4}$ Department of Internal Medicine and Liver Research Institute, Seoul National University College of Medicine, Seoul, Korea; ${ }^{5}$ Yonsei Plus Clinic, \\ Seongnam, Gyeonggi-do, Korea; 'Department of Periodontology, School of Dentistry, Wonkwang University, Iksan, Jeollabuk-do, Korea; \\ ${ }^{7}$ Department of Public Health, Graduate School of Chungang University, Seoul, Korea; ${ }^{8}$ Biomedical Research Institute, Seoul National University \\ Bundang Hospital, Gyeonggi-do, Korea; ${ }^{9}$ Medical Research Collaborating Center, Seoul National University Bundang Hospital, Gyeonggi-do, \\ Korea; and ${ }^{10}$ Department of Oral Medicine and Oral Diagnosis, School of Dentistry, Seoul National University, Seoul, Korea
}

\section{Background/Aims}

The relationship between halitosis and gastroesophageal reflux disease (GERD) remains controversial. The aim of this study was to investigate an association between subjective and objective halitosis and GERD.

\section{Methods}

The subjects were enrolled from participants who visited a health promotion center at Seoul National University Bundang Hospital. For diagnosis of halitosis, a questionnaire was requested, and volatile sulfur compounds (VSCs) were measured by Halimeter. Self-conscious halitosis was defined as halitosis perceived by himself or herself. Informed halitosis was defined as halitosis perceived by others. Objective halitosis was defined when mean VSCS values were $>100$ parts per billion. GERD was defined based on a questionnaire and endoscopy, including erosive esophagitis and non-erosive reflux disease (NERD).

Results

A total of 54 subjects (male:female $=33: 21$ ) with mean age of $46.0 \pm 11.4$ years were analyzed. The mean VSCs values were not significantly different between presence and absence of self-conscious halitosis $(P=0.322)$, but significantly different between presence and absence of informed halitosis $(P=0.021)$. Informed halitosis was associated with objective halitosis $(P=$ 0.039). GERD, erosive esophagitis and NERD did not correlate with objective halitosis $(P=0.556,0.206$ and 0.902 , respectively). In multivariable analysis, the relationship between objective halitosis and GERD symptoms including chest pain, heart burn, acid regurgitation, epigastric pain, hoarseness, globus sensation and coughing was not significant. Besides, GERD was not associated with self-conscious halitosis, informed halitosis and objective halitosis, respectively.

Received: April 18, 2014 Revised: May 30, 2014 Accepted: June 2, 2014

(c) This is an Open Access article distributed under the terms of the Creative Commons Attribution Non-Commercial License (http://creativecommons. org/licenses/by-nc/3.0) which permits unrestricted non-commercial use, distribution, and reproduction in any medium, provided the original work is properly cited.

*Correspondence: Nayoung Kim, MD, PhD

Department of Internal Medicine, Seoul National University Bundang Hospital, 173-82, Gumi-ro, Bundang-gu, Seongnam, Gyeonggi-do 463-707, Korea

Tel: +82-31-787-7008, Fax: +82-31-787-4051, E-mail: nayoungkim49@empas.com

Hyo-Jung Lee and Hee Man Kim equally contributed to this work.

Financial support: This study was supported by the R\&D fund of Seoul National University Bundang Hospital (No. 11-2011-002).

Conflicts of interest: None.

Author contributions: Hyo-Jung Lee and Hee Man Kim interpreted data and revised the manuscript; Nayoung Kim designed this study and supervised preparing manuscript; Jane C Oh checked the contents and edited English; Hyun Jin Jo checked the contents and revised the manuscript; Jung-Tae Lee and Hee-Young Chang collected data; Na-Hee Chang measured the Halitosis level; Soyeon Ahn analyzed data and drafted the article; Jeong-Yun Lee advised design.

ORCID: Nayoung Kim, http://orcid.org/0000-0002-9397-0406; 


\section{Conclusions}

GERD might not be associated with self-conscious, informed halitosis and objective halitosis indicated by Halimeter results. Informed halitosis could be correlated with objective halitosis determined by the Halimeter.

(J Neurogastroenterol Motil 2014;20:483-490)

\section{Key Words}

Halitosis; Gastroesophageal reflux; Questionnaires

\section{Introduction}

Halitosis is a general term used to define an unpleasant odor from the breath. Halitosis itself is not a serious illness but it frequently causes a very troublesome situation in interpersonal social relationship. ${ }^{1,2}$ It has become an important market for the pharmacological and cosmetic industries. ${ }^{3}$ The prevalence of halitosis varies according to study populations because the perception of halitosis differs among culturally diverse population. ${ }^{3}$ In Japan, population-based studies have reported that the prevalence of halitosis is $6-23 \%{ }^{4,5}$ In other countries, even more than $50 \%$ of the study population has been estimated to have halitosis. ${ }^{6}$

The causes of halitosis are mainly related to intraoral factors including microorganisms on the dorsal surface of tongue. ${ }^{7-11}$ Volatile sulfur compounds (VSCs) including hydrogen sulfide, methyl mercaptan and dimethyl sulfide are known as the main components of halitosis, and they are usually produced by degradation of amino acids by gram negative anaerobic oral bacteria. ${ }^{4,9,12}$ Non-oral factors of halitosis include pharyngotonsillar problems, problems of the gastrointestinal and respiratory system, liver cirrhosis, chronic renal failure, diabetes mellitus, even malignant disease, as well as imaginary halitosis such as pseudo-halitosis or halitophobia. ${ }^{5,13,14}$

Halitosis has been considered to be associated with the gastrointestinal system. As many as $41 \%$ of German dentists have believed that halitosis is caused mostly by stomach problems. ${ }^{5}$ Recently, gastroesophageal reflux disease (GERD) has been reported to be one of the causes of halitosis. ${ }^{15-19}$ Moreover a study has reported that GERD-related symptoms were associated with halitosis, regardless of the oral conditions. ${ }^{17}$ In contrast, some studies have reported that halitosis is not associated with erosive GERD. $^{20,21}$ The relationship between halitosis and GERD therefore remains controversial.

The diagnostic method of halitosis has not been standardized, and there is no consensus of diagnostic criteria for halitosis.
So far, the diagnosis of halitosis has been mainly dependent on subjective self-reported questionnaires developed by various researchers. ${ }^{17-19}$ A Halimeter (RH-17K; Interscan Co., Chatsworth, CA, USA) has been introduced for diagnosis of halitosis by detecting VSCs, and is considered as an objective test for halitosis. The aim of this study was to investigate an association between GERD and halitosis diagnosed by self-reported questionnaires and VSCs measurements by the Halimeter.

\section{Materials and Methods}

\section{Subjects}

The study subjects were collected from the participants who had visited the Health Promotion Center at Seoul National University Bundang Hospital, Seongnam, Republic of Korea. This examination program included anthropometric measurement, a blood test, X-ray and esophagogastroduodenoscopy. Prior to the commencement of this study, all subjects were given an explanation of the study and signed informed consent forms. The subjects were requested to complete 3 types of questionnaires: (1) lifestyle habits and comorbidities, (2) halitosis questionnaire and (3) symptoms of gastroesophageal reflux. The participants who did not receive some part of health examination or did not complete a questionnaire were excluded. The participants whose values of VSCs by the Halimeter were $>500$ parts per billion (ppb) or $<10$ ppb were excluded. This study protocol was approved by the Institutional Review Board of Seoul National University Bundang Hospital (No. B-1102/122-301).

\section{Halitosis Assessment}

\section{Halitosis questionnaire}

The halitosis questionnaire was developed to assess subjective halitosis. The questionnaire includes questions about halitosis-related themes: presence of self-perceived halitosis, presence of hal- 
itosis perceived by others, frequency, onset and duration of halitosis, oral conditions, and oral hygiene. The questions of the questionnaire are as follows: (1) Can you detect your bad breath? (a. yes, b. no) (2) How did you know that you have bad breath? (a. I know myself, b. I guess from other's comment. c. I think only I can detect my bad breath) (3) Have you ever been shocked by other people's reaction to your bad breath? (a. yes, b. no) (4) Do you have sinus infection? (a. yes, b. no) (5) Do you have rhinitis? (a. yes, b. no).

\section{Volatile sulfur compounds measurement using Halimeter}

The concentration of VSCs was measured by Halimeter (RH-17K; Interscan Co., Chatsworth, CA, USA). ${ }^{22-25}$ The measurement of VSCs was performed in the morning (from 8 AM to11 AM). All subjects refrained from any oral activity such as food intake for at least 2 hours prior to the measurement. The subjects were instructed to close their mouths for 3 minutes, breathing only through the nose before every measurement, followed by a straw connected to the Halimeter being placed at $4 \mathrm{~cm}$ into their mouth. The measurement was done automatically and the mean value of consecutive 3-time measures was used for analysis. $^{8}$

\section{Definition}

Subjective halitosis was defined as 2 types of halitosis: self-conscious halitosis and informed halitosis. Subsequently, self-conscious halitosis was defined as halitosis perceived by himself or herself. Informed halitosis was defined as halitosis when he or she was told by others that he or she had a malodor from his or her mouth. Objective halitosis was defined as $>100 \mathrm{ppb}$ of VSCs concentration measured by the Halimeter. ${ }^{9,11,26}$ GERD included non-erosive reflux disease (NERD) and erosive esophagitis with or without gastroesophageal reflux symptoms surveyed by a questionnaire. Erosive esophagitis was defined based on the endoscopic findings according to the Los Angeles classification. NERD was diagnosed when a subject responded that reflux symptoms were present at a frequency of at least once per week in the absence of erosive esophagitis. ${ }^{27}$ The questions of the GERD questionnaire are as follows ${ }^{27,28}$ : (1) Have you experienced heartburn (a. soreness in the substernal area, b. a burning sensation or discomfort in the substernal area, c. sensation of salt pepper in the epigastric area or $\mathrm{d}$. a burning sensation induced by water swallowing) within the past year? (2) Have you experienced acid regurgitation (a. sour water brash in the mouth or throat, b. a sense of food regurgitation) within the past year? (3) Have you experienced chest pain within the past year? (4) Have you experienced hoarseness within the past year? (5) Have you experienced a globus sensation in the throat (a. foreign body sensation in the throat, b. sticky food sensation in the epigastric area) within the past year? (6) Have you experienced a frequent cough within the past year? (7) Have you experienced epigastric soreness within the past year? The subjects who responded positively to any one of these 7 symptoms were asked to choose the most bothersome symptom. In addition, the frequency (a. 1-2 times per year, b. 1-2 times per month, c. 1-2 times per week, d. 3-4 times per week, e. daily) and severity (a. mild; b. moderate, bothersome to everyday life; c. severely disturbing work or sleeping) of the most bothersome symptom was determined. In addition, subjects were asked whether they had taken histamine type $2(\mathrm{H} 2)$ receptor blocker or proton pump inhibitor (PPI) to relieve these symptoms.

\section{Statistical Methods}

The statistical analysis was performed using SPSS software (SPSS version 18.0, Chicago, IL, USA). Student's $t$ test was used to compare means. The Chi-square test and Fisher's exact test were used to investigate relationships among factors. Multivariable analysis was performed using a logistic regression model. A value of less than $P<0.05$ was considered to indicate a statistically significant difference.

\section{Results}

Between July 2011 and August 2012, a total of 93 subjects who had visited the Health Promotion Center were enrolled. Of them, 34 subjects were excluded because they did not receive upper GI endoscopy, and 5 patients were excluded because they had extreme values of VSCs ( $>500 \mathrm{ppb}$ or $<10 \mathrm{ppb}$ ). A total of 54 subjects including $33(61.1 \%)$ male and 21 (38.9\%) female were analyzed in this study, and the mean age was $46.0 \pm 11.4$ years (Table 1). The mean values of VSCs did not differ according to the status of obesity, alcohol intake, smoking, rhinitis and sinusitis $(P=0.068,0.923,0.209,0.625$ and 0.623 , respectively). The subjects with diabetes mellitus had higher levels of VSCs than those without diabetes mellitus $(329.4 \pm 205.3$ ppb vs. $132.2 \pm$ $88.5 \mathrm{ppb}, P=0.034)$. The number of subject with self-conscious halitosis was $30(55.5 \%)$ and the number of subject with informed halitosis was $12(22.2 \%)$. The mean VSCs value was not significantly different between presence and absence of self-conscious halitosis $(P=0.322)$. The presence of informed halitosis showed higher values of VSCs than the absence of informed hal- 
Table 1. Baseline Characteristics of All Subjects $(\mathrm{N}=54)$

\begin{tabular}{|c|c|c|c|c|c|}
\hline & \multirow{2}{*}{$\begin{array}{c}\text { Halimeter } \\
(\text { mean } \pm \mathrm{SD}, \mathrm{ppb})\end{array}$} & \multirow{2}{*}{$P$-value } & \multicolumn{2}{|c|}{ Halimeter } & \multirow{2}{*}{$P$-value } \\
\hline & & & $>100 \mathrm{ppb}(\mathrm{n}[\%])$ & $\leq 100 \mathrm{ppb}(\mathrm{n}[\%])$ & \\
\hline Number of subjects & 54 & & 31 & 23 & \\
\hline Age (mean $\pm \mathrm{SD}, \mathrm{yr})$ & $46.0 \pm 11.4$ & & $44.71 \pm 10.3$ & $47.78 \pm 12.7$ & 0.127 \\
\hline Gender & & 0.684 & & & 0.246 \\
\hline Male & $155.9 \pm 115.8$ & & $21(67.7)$ & $12(52.2)$ & \\
\hline Female & $141.8 \pm 120.2$ & & $10(32.3)$ & $11(47.8)$ & \\
\hline Body mass index & & 0.675 & & & 0.462 \\
\hline$\geq 25 \mathrm{~kg} / \mathrm{m}^{2 \mathrm{a}}$ & $160.1 \pm 129.3$ & & $11(35.5)$ & $6(26.1)$ & \\
\hline$<25 \mathrm{~kg} / \mathrm{m}^{2}$ & $146.0 \pm 111.9$ & & $20(64.5)$ & $17(73.9)$ & \\
\hline Alcohol consumption & & 0.923 & & & 0.836 \\
\hline Yes & $137.0 \pm 91.0$ & & $18(58.1)$ & $14(60.9)$ & \\
\hline No & $170.0 \pm 146.3$ & & $13(41.9)$ & $9(39.1)$ & \\
\hline Smoking & & 0.209 & & & 0.319 \\
\hline Yes $^{\mathrm{b}}$ & $181.8 \pm 128.9$ & & $8(25.8)$ & $3(13.0)$ & \\
\hline No & $142.4 \pm 113.5$ & & $23(74.2)$ & $20(87.0)$ & \\
\hline Diabetes Mellitus & & 0.034 & & & 0.380 \\
\hline Yes & $329.4 \pm 205.3$ & & $4(12.9)$ & $1(4.3)$ & \\
\hline No & $132.2 \pm 88.5$ & & $27(87.1)$ & $22(95.7)$ & \\
\hline Rhinitis & & 0.625 & & & 0.462 \\
\hline Yes & $169.5 \pm 124.8$ & & $11(35.5)$ & $6(26.1)$ & \\
\hline No & $141.7 \pm 113.3$ & & $20(64.5)$ & $17(73.9)$ & \\
\hline Sinusitis & & 0.623 & & & 0.273 \\
\hline Yes & $172.2 \pm 98.1$ & & $7(22.6)$ & $2(8.7)$ & \\
\hline No & $146.1 \pm 120.5$ & & $24(77.4)$ & $21(91.3)$ & \\
\hline Self-conscious halitosis & & 0.322 & & & 0.667 \\
\hline Yes & $166.7 \pm 131.0$ & & $18(58.1)$ & $12(52.2)$ & \\
\hline No & $130.1 \pm 94.4$ & & $13(41.9)$ & $11(47.8)$ & \\
\hline Informed halitosis & & 0.021 & & & 0.039 \\
\hline Yes & $215.6 \pm 128.1$ & & $10(32.3)$ & $2(8.7)$ & \\
\hline No & $131.8 \pm 107.6$ & & $21(67.7)$ & $21(91.3)$ & \\
\hline GERD & & 0.126 & & & 0.556 \\
\hline Yes & $81.5 \pm 60.9$ & & $6(19.4)$ & $6(26.1)$ & \\
\hline No & $156.6 \pm 119.4$ & & $25(80.6)$ & $17(73.9)$ & \\
\hline Erosive esophagitis & & 0.787 & & & 0.206 \\
\hline Yes & $139.3 \pm 97.6$ & & $2(6.5)$ & $4(17.4)$ & \\
\hline No & $149.6 \pm 121.0$ & & $29(93.5)$ & $19(82.6)$ & \\
\hline NERD & & 0.963 & & & 0.902 \\
\hline Yes & $152.1 \pm 103.1$ & & $5(16.1)$ & $4(17.4)$ & \\
\hline No & $150.1 \pm 120.2$ & & $26(83.9)$ & $19(82.6)$ & \\
\hline
\end{tabular}

abesity in Korean is defined as $\geq 25 \mathrm{~kg} / \mathrm{m}^{2}$ in body mass index, ${ }^{\mathrm{b}}$ Current and former.

GERD, gastroesophageal reflux disease; NERD, non-erosive reflux disease.

itosis $(P=0.021)$, and informed halitosis correlated with objective halitosis diagnosed by Halimeter $(P=0.039)$. GERD, erosive esophagitis and NERD were not significantly associated with objective halitosis ( $P=0.556,0.206$ and 0.902 , respectively).

GERD symptoms, including chest pain, heartburn, acid re- gurgitation, epigastric soreness, hoarseness, globus sensation and coughing, were not significantly associated with objective halitosis (Table 2). In multivariable analysis, each of GERD symptoms was not significantly associated with objective halitosis (Table 2).

In multivariable analysis, self-conscious halitosis was not sig- 
Table 2. Association Between Symptoms of Gastroesophageal Reflux Disease and Halimeter

\begin{tabular}{|c|c|c|c|c|c|c|}
\hline & \multicolumn{3}{|c|}{ Halimeter } & \multicolumn{3}{|c|}{ Halimeter $(>100 \mathrm{ppb})$} \\
\hline & $\begin{array}{c}>100 \mathrm{ppb} \\
(\mathrm{n}=31, \mathrm{n}[\%])\end{array}$ & $\begin{array}{c}\leq 100 \mathrm{ppb} \\
(\mathrm{n}=23, \mathrm{n}[\%])\end{array}$ & $P$-value & $\mathrm{OR}^{\mathrm{a}}$ & $95 \% \mathrm{CI}$ & $P$-value \\
\hline Chest pain & & & 0.217 & & & 0.369 \\
\hline Yes & $3(9.7)$ & $5(21.7)$ & & 0.48 & $0.01-2.39$ & \\
\hline No & $28(90.3)$ & $18(78.3)$ & & & & \\
\hline Heartburn & & & 0.717 & & & 0.120 \\
\hline Yes & $6(19.4)$ & $3(13.0)$ & & 3.19 & $0.74-13.75$ & \\
\hline No & $25(80.6)$ & $20(87.0)$ & & & & \\
\hline Acid regurgitation & & & 0.529 & & & 0.762 \\
\hline Yes & $12(38.7)$ & $7(30.4)$ & & 1.20 & $0.37-3.94$ & \\
\hline No & $19(61.3)$ & $16(69.6)$ & & & & \\
\hline Epigastric soreness & & & 0.981 & & & 0.776 \\
\hline Yes & $8(25.8)$ & $6(26.1)$ & & 0.83 & $0.23-3.03$ & \\
\hline No & $23(74.2)$ & $17(73.9)$ & & & & \\
\hline Hoarseness & & & 0.218 & & & 0.123 \\
\hline Yes & $6(19.4)$ & $1(4.3)$ & & 5.28 & $0.62-54.55$ & \\
\hline No & $25(80.6)$ & $22(95.7)$ & & & & \\
\hline Globus sensation & & & 0.443 & & & 0.410 \\
\hline Yes & $3(9.7)$ & $4(17.4)$ & & 0.51 & $0.10-2.54$ & \\
\hline No & $28(90.3)$ & $19(82.6)$ & & & & \\
\hline Coughing & & & 0.741 & & & 0.640 \\
\hline Yes & $7(22.6)$ & $4(17.4)$ & & 1.39 & $0.35-5.44$ & \\
\hline No & $24(77.4)$ & $19(82.6)$ & & & & \\
\hline
\end{tabular}

${ }^{\mathrm{a}}$ Adjustment for age and gender.

Table 3. Multivariable Analysis Association Between Halitosis and Gastroesophageal Reflux Disease

\begin{tabular}{|c|c|c|c|c|c|c|c|c|c|}
\hline & \multicolumn{3}{|c|}{ Self-conscious halitosis } & \multicolumn{3}{|c|}{ Informed halitosis } & \multicolumn{3}{|c|}{ Halimeter $(>100 \mathrm{ppb})$} \\
\hline & OR & $95 \% \mathrm{CI}$ & $P$-value & OR & $95 \% \mathrm{CI}$ & $P$-value & OR & $95 \% \mathrm{CI}$ & $P$-value \\
\hline GERD & 3.00 & $0.56-16.14$ & 0.201 & 0.55 & $0.09-3.54$ & 0.530 & 0.69 & $0.17-2.78$ & 0.598 \\
\hline Age $(\geq 45 \mathrm{yr})$ & 0.41 & $0.11-1.46$ & 0.167 & 7.11 & $1.11-45.60$ & 0.039 & 0.90 & $0.27-3.05$ & 0.865 \\
\hline Gender (male) & 0.24 & $0.05-1.24$ & 0.042 & 0.88 & $0.13-6.16$ & 0.898 & 2.29 & $0.48-10.90$ & 0.297 \\
\hline Obesity & 1.90 & $0.37-9.82$ & 0.442 & 4.68 & $0.83-26.47$ & 0.081 & 1.30 & $0.32-5.32$ & 0.713 \\
\hline Smoking & 6.52 & $1.07-39.63$ & 0.042 & 2.00 & $0.28-14.33$ & 0.489 & 1.76 & $0.32-9.85$ & 0.519 \\
\hline Alcohol & 0.43 & $0.10-1.95$ & 0.274 & 0.44 & $0.07-2.76$ & 0.383 & 0.38 & $0.09-1.70$ & 0.208 \\
\hline Rhinitis & 2.81 & $0.43-18.38$ & 0.281 & 3.36 & $0.49-23.22$ & 0.219 & 1.38 & $0.30-6.27$ & 0.678 \\
\hline Sinusitis & 0.54 & $0.06-4.99$ & 0.586 & 1.55 & $0.17-13.99$ & 0.697 & 2.89 & $0.37-22.49$ & 0.310 \\
\hline
\end{tabular}

GERD, gastroesophageal reflux disease.

Multivariable analysis using logistic regression model: age, gender, smoking, alcohol, rhinitis and sinusitis.

nificantly associated with GERD (odds ratio [OR], 3.00; 95\% confidence interval [CI], 0.56-16.14) (Table 3). Smoking was strongly associated with self-conscious halitosis $(6.52 ; 95 \% \mathrm{CI}$, 1.07-39.63). Informed halitosis was not significantly associated with GERD (OR, 0.55; 95\% CI, 0.09-3.54). Old age was strongly associated with informed halitosis (OR, 7.11; 95\% CI,
1.11-45.60). Objective halitosis was not significantly associated with GERD (OR, 0.69; 95\% CI, 0.17-2.78).

\section{Discussion}

This study showed that GERD was not significantly asso- 


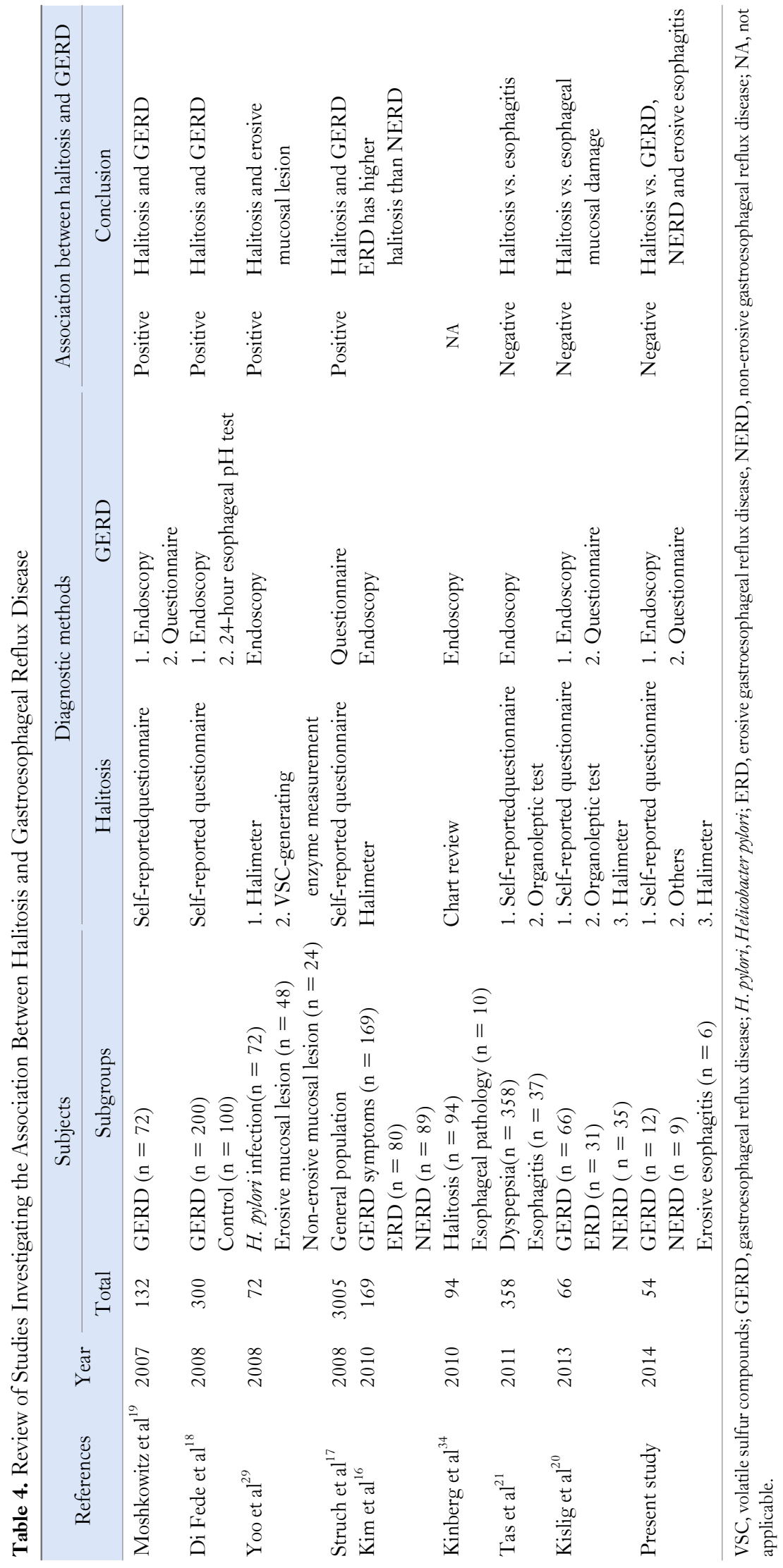


ciated with self-conscious halitosis, informed halitosis and objective halitosis, respectively. In addition, individual symptoms of GERD were not associated objective halitosis. Only smoking and old age were significantly associated with self-conscious halitosis and informed halitosis, respectively.

The relationship between halitosis and GERD is controversial. Several studies have reported no significant association which is consistent with our findings (Table 4). The results of our study seem to match up with the previous studies which did not suggest scientific evidences for the causal relationship between halitosis and GERD, ${ }^{20,21}$ and even inferring GERD as an extra-oral factor to play only a minor role as a potential cause for halitosis., ${ }^{5,14}$

However, other studies have proposed a significant relationship between halitosis and GERD (Table 4). ${ }^{16-19,29}$ Di Fede et $\mathrm{al}^{18}$ have reported that patients with endoscopically detectable erosion exhibited higher levels of self-reported halitosis in comparison to controls. Moreover, Moshkowitz et $\mathrm{al}^{19}$ have reported that subjective halitosis may be considered as an extra-esophageal manifestation of GERD. Struch et $\mathrm{al}^{17}$ have found a positive relationship between self-reported symptoms of GERD and self-reported halitosis in a German population. However, self-reported halitosis does not always correlate with objective findings of halitosis, such as VSC levels. ${ }^{1,430}$ Two Korean studies investigated the relationship between halitosis by Halimeter results and GERD including erosive reflux disease and NERD. ${ }^{16,29}$ In these studies, the erosive reflux disease group had higher VSC values than the NERD group. However, these studies lacked a true control group, such as healthy subjects. The exact mechanism how acid reflux causes high VSCs in oral cavity in GERD patients remains unclear although GERD could be associated with halitosis.

Awareness of halitosis is not always consistent with the actual presence of halitosis. In our study, self-conscious halitosis was not associated with objective halitosis, but informed halitosis was associated with objective halitosis. This phenomenon is explained by the "bad breath paradox." ${ }^{8,14,17}$ Individuals with objective halitosis can be unaware of the problem, whereas others are convinced that those individuals have halitosis even when no objective evidence are found. ${ }^{17,31-33}$ In addition, some proportion of the latter ones may be considered to have pseudo-halitosis or halitophobia. $^{5,8,11}$

Our study has several limitations. First, an objective examination about intra-oral conditions such as tongue coating and the presence of periodontal disease was not performed. Quirynen et $\mathrm{al}^{14}$ have suggested that tongue coating (43\%) is the most fre- quent associated halitosis factor, followed by a combination of tongue coating, periodontitis and gingivitis (18.2\%) and periodontal disease $(11.1 \%)$ as the sole underlying factor. However, one study by Kislig et $\mathrm{al}^{20}$ have reported that GERD is not associated with objective tongue coating, and therefore intra-oral conditions may not be a critical factor to affect the association between halitosis and GERD. Second, the sample size was relatively small. Originally, 93 subjects were enrolled, but 39 subjects were excluded unexpectedly. We tested the differences of clinical features between 93 subjects and 54 subjects. The mean age, the mean VSCs values and the prevalence of GERD symptoms were not statistically different between them, but the portion of male subjects were significantly more in 54 subjects than in 93 subjects (61.1\% vs. $44.1 \%, P=0.047)$. In our results, male gender did not have any effect on halitosis, but the interpretation should be cautious due to the difference in gender between the initial enrolled subjects $(\mathrm{n}=93)$ and analyzed subjects $(\mathrm{n}=54)$. Third, the intake of medicines like $\mathrm{H} 2$ receptor blocker and PPI were not controlled. The effect of acid suppressants on halitosis remains unclear, but it might affect halitosis if reflux of gastric acid would be associated with halitosis. The effect of $\mathrm{H} 2$ receptor blocker and PPI on halitosis should be investigated.

In conclusion, this study shows that GERD is not associated with halitosis diagnosed by a self-reported questionnaire and by Halimeter. These suggest that acid reflux may not affect halitosis. In addition, Halimeter correlates more with informed halitosis than self-conscious halitosis, and therefore Halimeter is a reliable diagnostic method of halitosis.

\section{Acknowledgements}

The authors would like to express their gratitude to Medical Research Collaborating Center of Seoul National University Bundang Hospital for their assistance in statistical analysis. The authors are indebted to Professor Emeritus J Patrick Barron (Tokyo Medical University, Tokyo, Japan and as Adjunct Professor, Seoul National University Bundang Hospital, Bundang, Republic of Korea) for his pro bono editing of this manuscript.

\section{References}

1. Rosenberg M. Clinical assessment of bad breath: current concepts. J Am Dent Assoc 1996;127:475-482.

2. Bosy A. Oral malodor: philosophical and practical aspects. J Can Dent Assoc 1997;63:196-201.

3. Scully C, Greenman J. Halitosis (breath odor). Periodontol 2000 
2008;48:66-75

4. Miyazaki H, Sakao S, Katoh Y, Takehara T. Correlation between volatile sulphur compounds and certain oral health measurements in the general population. J Periodontol 1995;66:679-684.

5. Seemann R, Bizhang M, Djamchidi C, Kage A, Nachnani S. The proportion of pseudo-halitosis patients in a multidisciplinary breath malodour consultation. Int Dent J 2006;56:77-81.

6. Tessier JF, Kulkarni GV. Bad breath: etiology, diagnosis and treatment. Oral Health 1991;81:19-22, 24.

7. Tonzetich J. Oral malodour: an indicator of health status and oral cleanliness. Int Dent J 1978;28:309-319.

8. Delanghe G, Ghyselen J, van Steenberghe D, Feenstra L. Multidisciplinary breath-odour clinic. Lancet 1997;350:187.

9. Porter SR, Scully C. Oral malodour (halitosis). BMJ 2006;333:632635.

10. Tangerman A, Winkel EG. Intra- and extra-oral halitosis: finding of a new form of extra-oral blood-borne halitosis caused by dimethyl sulphide. J Clin Periodontol 2007;34:748-755.

11. Bollen CM, Beikler T. Halitosis: the multidisciplinary approach. Int J Oral Sci 2012;4:55-63.

12. Tonzetich J, Richter VJ. Evaluation of volatile odoriferous components of saliva. Arch Oral Biol 1964;9:39-46.

13. Eli I, Baht R, Koriat H, Rosenberg M. Self-perception of breath odor. J Am Dent Assoc 2001;132:621-626.

14. Quirynen M, Dadamio J, Van den Velde S, et al. Characteristics of 2000 patients who visited a halitosis clinic. J Clin Periodontol 2009; 36:970-975.

15. Lee H, Kho HS, Chung JW, Chung SC, Kim YK. Volatile sulfur compounds produced by Helicobacter pylori. J Clin Gastroenterol 2006; 40:421-426.

16. Kim JG, Kim YJ, Yoo SH, et al. Halimeter ppb Levels as the predictor of erosive gastroesophageal reflux disease. Gut Liver 2010;4: 320-325.

17. Struch F, Schwahn C, Wallaschofski H, et al. Self-reported halitosis and gastro-esophageal reflux disease in the general population. J Gen Int Med 2008;23:260-266.

18. Di Fede O, Di Liberto C, Occhipinti G, et al. Oral manifestations in patients with gastro-oesophageal reflux disease: a single-center casecontrol study. J Oral Pathol Med 2008;37:336-340.

19. Moshkowitz M, Horowitz N, Leshno M, Halpern Z. Halitosis and gastroesophageal reflux disease: a possible association. Oral Dis 2007; 13:581-585.

20. Kislig K, Wilder-Smith CH, Bornstein MM, Lussi A, Seemann R. Halitosis and tongue coating in patients with erosive gastroesopha- geal reflux disease versus nonerosive gastroesophageal reflux disease. Clin Oral Investig 2013;17:159-165.

21. Tas A, Köklü S, Yüksel I, Basar O, Akbal E, Cimbek A. No significant association between halitosis and upper gastrointestinal endoscopic findings: a prospective study. Chin Med J 2011;124:37073710.

22. Murata T, Fujiyama Y, Yamaga T, Miyazaki H. Breath malodor in an asthmatic patient caused by side-effects of medication: a case report and review of the literature. Oral Dis 2003;9:273-276.

23. Moayyedi P, Talley NJ. Gastro-oesophageal reflux disease. Lancet 2006;367:2086-2100.

24. Rosenberg M, McCulloch CA. Measurement of oral malodor: current methods and future prospects. J Periodontol 1992;63:776-782.

25. Cho YK, Kim GH, Kim JH, et al. Diagnosis of gastroesophageal reflux disease: a systematic review. Korean J Gastroenterol 2010;55: 279-295.

26. Snel J, Burgering M, Smit B, et al. Volatile sulphur compounds in morning breath of human volunteers. Arch Oral Biol 2011;56:29-34.

27. Kim N, Lee SW, Cho SI, et al. The prevalence of and risk factors for erosive oesophagitis and non-erosive reflux disease: a nationwide multicentre prospective study in Korea. Aliment Pharmacol Ther 2008;27:173-185.

28. Kim JY, Kim N, Seo PJ, et al. Association of sleep dysfunction and emotional status with gastroesophageal reflux disease in Korea. J Neurolgastroenterol Motil 2013;19:344-354.

29. Yoo SH, Jung HS, Sohn WS, et al. Volatile sulfur compounds as a predictor for esophagogastroduodenal mucosal injury. Gut Liver 2008;2:113-118.

30. van den Broek AM, Feenstra L, de Baat C. A review of the current literature on aetiology and measurement methods of halitosis. J Dent 2007;35:627-635.

31. Nasseri-Moghaddam S, Nokhbeh-Zaeem H, Saniee P, Pedramnia S, Sotoudeh M, Malekzadeh R. Oral nitrate reductase activity and erosive gastro-esophageal reflux disease: a nitrate hypothesis for GERD pathogenesis. Dig Dis Sci 2012;57:413-418.

32. Delanghe G, Ghyselen J, Bollen C, van Steenberghe D, Vandekerckhove BN, Feenstra L. An inventory of patients' response to treatment at a multidisciplinary breath odor clinic. Quintessence Int 1999;30: 307-310.

33. Rosenberg M, Kozlovsky A, Gelernter I, et al. Self-estimation of oral malodor. J Dent Res 1995;74:1577-1582.

34. Kinberg S, Stein M, Zion N, Shaoul R. The gastrointestinal aspects of halitosis. Can J Gastroenterol 2010;24:552-556. 